\title{
RESEARCH
}

\section{Corrigendum to: The asymptotic profile of an eta-theta quotient related to entanglement entropy in string theory}

\author{
Joshua Males*(i)
}

${ }^{*}$ Correspondence: jmales@math.uni-koeln.de Department of Mathematics and Computer Science, University of Cologne, Weyertal 86-90, 50931 Cologne, Germany

The original article can be found online at https://doi.org/10. 1007/s40993-020-0190-x

\section{Abstract \\ This corrigendum serves to correct the article [1, Theorem 1.1]. In doing so, we correct the proof and statement of Theorem 3.7, and see that one may disregard Proposition 3.4, Lemma 3.5 and Proposition 3.6.}

\section{Corrigendum to: J. Males Res. Number Theory (2020) 6:15 https://doi.org/10.1007/s40993-020-0190-x}

This corrigendum deals with two issues in the article [1] kindly pointed out to the author by S. Zwegers. There is an error that occurs in the use of the integrals $G^{+}$and $G^{-}$, and another in the treatment of the functions $g_{m, j}$ for $j=1,2,3$ where exponential growth should occur. Overall, these do not greatly impact the main results of the paper. In particular, Theorem 1.1 holds up to slight changes in constants and the correct version is stated as follows.

Theorem 1.1 For $\beta:=\pi \sqrt{\frac{2}{n}}$ and $|m| \leq \frac{1}{6 \beta} \log (n)$ we have that

$$
b(m, n)=(-1)^{m+\delta+\frac{3}{2}} \frac{\beta^{6} m}{8 \pi^{5}(2 n)^{\frac{1}{4}}} e^{2 \pi \sqrt{2 n}}+O\left(m n^{-\frac{15}{4}} e^{2 \pi \sqrt{2 n}}\right)
$$

as $n \rightarrow \infty$. Here, $\delta:=1$ if $m<0$ and $\delta=0$ otherwise.

In the paper, it is claimed that $G^{+}=G^{-}$, which gives rise to the residue term. However, this is not the case. In fact, $G^{+}=-G^{-}$, and so the final term appearing in equation (3.1) vanishes. Instead, we have that

$$
f_{m}(\tau):=\frac{f_{m}^{+}+f_{m}^{-}}{2}=-2 i \int_{0}^{\frac{1}{2}} f(z ; \tau) \sin (2 \pi m z) d z
$$

which can be easily seen by mapping $z \mapsto 1-z$ on the path of integration. One does not require the limit as $a \rightarrow 0^{+}$, as the integrand is well-defined for all $z \in\left[0, \frac{1}{2}\right]$.

(c) The Author(s) 2021. This article is licensed under a Creative Commons Attribution 4.0 International License, which permits use, sharing, adaptation, distribution and reproduction in any medium or format, as long as you give appropriate credit to the original author(s) and the source, provide a link to the Creative Commons licence, and indicate if changes were made. The images or other third party material in this article are included in the article's Creative Commons licence, unless indicated otherwise in a credit line to the material. If material is not included in the article's Creative Commons licence and your intended use is not permitted by statutory regulation or exceeds the permitted use, you will need to obtain permission directly from the copyright holder. To view a copy of this licence, visit http://creativecommons.org/licenses/by/4.0/. 
Instead of the functions $g_{m, j}$, now one uses Lemma 3.1, which gives the asymptotic behaviour of $f$ for $0<z<\frac{1}{2}$. With this in hand, evaluating the sinh functions clearly shows that we have

$$
-\frac{\varepsilon^{3}}{\pi^{3}} \frac{\sinh \left(\frac{2 \pi^{2} z}{\varepsilon}\right)^{4}}{\sinh \left(\frac{4 \pi^{2} z}{\varepsilon}\right)}=-\frac{\varepsilon^{3}}{\pi^{3}} e^{\frac{4 \pi^{2} z}{\varepsilon}}\left(1+O\left(e^{-\frac{4 \pi^{2} z}{\varepsilon}}\right)\right) .
$$

Plugging in the formulae for the sinh functions and integrating explicitly, using the formula

$$
\int_{0}^{\frac{1}{2}} e^{x z} \sin (2 \pi m z) d z=-\frac{2 \pi m}{4 \pi^{2} m^{2}+x^{2}} e^{\frac{x}{2}} \cos (\pi m)-\frac{2 \pi m}{4 \pi^{2} m^{2}+x^{2}}
$$

shows directly that

$$
f_{m}(\tau)=-2 \frac{\varepsilon^{3}}{\pi^{3}}(-1)^{m+\frac{1}{2}} \frac{2 \pi m}{\left(\frac{16 \pi^{2}}{\varepsilon^{2}}\right)} e^{\frac{2 \pi^{2}}{\varepsilon}}+O\left(\varepsilon^{3}\right)=(-1)^{m+\frac{3}{2}} \frac{m}{4 \pi^{4}} \varepsilon^{5} e^{\frac{2 \pi^{2}}{\varepsilon}}+O\left(\varepsilon^{3}\right) .
$$

This replaces the statement given in Theorem 3.7. One then no longer requires the integrals $g_{m, j}$ for $j=1,2,3$, and as such can disregard Proposition 3.4, Lemma 3.5 and Proposition 3.6. The subsequent results in the paper hold, and in particular the main contribution to asymptotics is still given by the function at the dominant pole $q=1$.

Tracing the changes in constants to the use of the circle method yields Theorem 1.1.

Funding Open Access funding enabled and organized by Projekt DEAL.

Received: 16 April 2021 Accepted: 21 April 2021 Published online: 19 May 2021

References

1. Males, J.: The asymptotic profile of an eta-theta quotient related to entanglement entropy in string theory. Res. Number Theory 6(1), Paper No. 15, 14 (2020)

\section{Publisher's Note}

Springer Nature remains neutral with regard to jurisdictional claims in published maps and institutional affiliations. 